June 22, 1998

\title{
A Canonical Quantization of the Baker's Map*
}

\author{
Ron Rubin \\ Department of Mathematics \\ Massachusetts Institute of Technology \\ and the University of the Middle East Project \\ Email: rubin@math.mit.edu \\ Nathan Salwen \\ Lyman Laboratory of Physics \\ Harvard University \\ Email: salwen@fas.harvard.edu
}

\begin{abstract}
We present here a canonical quantization for the baker's map. The method we use is guite different from that used in Balazs and Voros (ref. and Saraceno (ref.2). We first construct a natural "baker covering map" on the plane $\mathbb{R}^{2}$. We then use as the quantum algebra of observables the subalgebra of operators on $L^{2}(\mathbb{R})$ generated by $\{\exp (2 \pi i \widehat{x}), \exp (2 \pi i \widehat{p})\}$. We construct a unitary propagator such that as $\hbar \rightarrow 0$ the classical dynamics is returned. For Planck's constant $h=1 / N$, we show that the dynamics can be reduced to the dynamics on an $N$-dimensional Hilbert space, and the unitary $N \times N$ matrix propagator is the same as given in ref. 1 except for a small correction of order $h$. This correction is shown to preserve the classical symmetry $x \rightarrow 1-x$ and $p \rightarrow 1-p$ in the quantum dynamics for periodic boundary conditions.
\end{abstract}

${ }^{*} 30$ pages, 2 figures, 1 table 


\section{Introduction}

The classical baker's map is a mapping of the unit torus onto itself defined as follows. Let $x$ and $p$ be the coordinates on the torus and take

$$
(x, p) \rightarrow\left(x^{\prime}, p^{\prime}\right)= \begin{cases}(2 x, p / 2), & 0 \leq x<1 / 2 \\ (2 x-1, p / 2+1 / 2), & 1 / 2 \leq x<1\end{cases}
$$

This map describes a stretching in $x$, shrinking in $p$, and chopping and stacking, similar to the way bakers make certain pastries. (See Figure 1.) The motion on the torus is completely chaotic with a positive Liapunov exponent $\log 2$, and is in fact a paradigm for the study of classical chaos. For more details on the classical baker's map, including a description of the map as a dynamics on binary digits, we refer the reader to ref. 1

A quantum version of the map was introduced by Balazs and Voros (ref. 4 ) and then by Saraceno (ref.2). In this description, the dynamics is quantized for values of Planck's constant satisfying $h=1 / N$, by constructing a quantum propagator $u_{m n}$ as a unitary $N \times N$ matrix. The classical limit is demonstrated numerically for $N \rightarrow \infty$. We present here a natural quantum propagator which, except for a correction of order $\hbar$, "reduces" to the finite dimensional matrix propagator given in ref. at the point $\theta=(0,0)$, corresponding to periodic boundary conditions of the quantum wave vectors defined below. This correction is what preserves the classical symmetry broken in the quantization scheme presented in ref. 10 Whether other points on the $\theta$-torus, including the anti-periodic case studied by Saraceno (ref.2), are invariant under the baker's map propagator derived below will be addressed in forthcoming papers (see ref.3).

\section{Baker's Map on the Plane}

Our quantization procedure uses a baker covering map we construct as folllows. The baker's map has a natural lift to the universal covering $\mathbb{R}^{2}$ of $\mathbb{T}^{2}$ given by

$$
\beta:(x, p) \rightarrow\left(x^{\prime}, p^{\prime}\right)= \begin{cases}(2 x, p / 2), & (x, p) \in l \cap e_{p} \\ (2 x-1, p / 2+1 / 2), & (x, p) \in r \cap e_{p} \\ (2 x+1, p / 2+1 / 2), & (x, p) \in l \cap o_{p} \\ (2 x, p / 2), & (x, p) \in r \cap o_{p}\end{cases}
$$


so that for $a, b \in \mathbb{Z}$,

$$
\beta^{*} e^{2 \pi i(a x+b p)}=e^{4 \pi i a x} e^{i \pi b p}\left(\chi_{l}(x)+(-1)^{b} \chi_{r}(x)\right)\left(\chi_{e_{p}}(p)+(-1)^{b} \chi_{o_{p}}(p)\right),
$$

where

$$
\begin{aligned}
l: & =\langle[0,1 / 2)+\mathbb{Z}\rangle \times \mathbb{R}, \\
r: & =\langle[1 / 2,1)+\mathbb{Z}\rangle \times \mathbb{R}, \\
e_{p}: & =\mathbb{R} \times\langle[0,1)+2 \mathbb{Z}\rangle, \\
o_{p}: & =\mathbb{R} \times\langle[1,2)+2 \mathbb{Z}\rangle .
\end{aligned}
$$

and

$$
\begin{aligned}
\chi_{l}(x) & =\left\{\begin{array}{ll}
1, & x \in[0,1 / 2)+\mathbf{Z} \\
0, & \text { otherwise }
\end{array},\right. \\
\chi_{r}(x) & =\left\{\begin{array}{ll}
1, & x \in[1 / 2,1)+\mathbf{Z} \\
0, & \text { otherwise }
\end{array},\right. \\
\chi_{e_{p}}(p) & =\left\{\begin{array}{ll}
1, & p \in[0,1)+2 \mathbf{Z} \\
0, & \text { otherwise }
\end{array},\right. \\
\chi_{o_{p}}(p) & = \begin{cases}1, & p \in[1,2)+2 \mathbf{Z} \\
0, & \text { otherwise }\end{cases}
\end{aligned}
$$

We can see what this covering map does to the plane in Figure 2. There are two important observations to make regarding this figure. The first is that every "LEFT" region gets mapped into a "BOTTOM" region and every "RIGHT" into a "TOP." Thus when the dynamics is given modulo 1, we return precisely the baker's map on the torus. The second is that there are, of course, many such natural covering maps. Another, for instance, would be to transport each fundamental domain to the first square $([0,1) \times[0,1))$, perform the baker transformation (eqn. 1), then shift back. The problem is this procedure requires a different algebraic form for the map in each fundamental domain of the plain. The benefit of the baker covering map presented here is there are only four fundamental regions required to write the map.

We can also write down the inverse of the baker covering map:

$$
\beta^{-1}:(x, p) \rightarrow\left(x^{\prime}, p^{\prime}\right)= \begin{cases}(x / 2,2 p), & (x, p) \in e_{x} \cap b ; \\ (x / 2-1 / 2,2 p-1), & (x, p) \in o_{x} \cap b ; \\ (x / 2+1 / 2,2 p-1), & (x, p) \in e_{x} \cap t \\ (x / 2,2 p), & (x, p) \in o_{x} \cap t\end{cases}
$$


where we have used the "conjugate" regions

$$
\begin{aligned}
b: & =\mathbb{R} \times\langle[0,1 / 2)+\mathbb{Z}\rangle, \\
t: & =\mathbb{R} \times\langle[1 / 2,1)+\mathbb{Z}\rangle, \\
e_{x}: & =\langle[0,1)+2 \mathbb{Z}\rangle \times \mathbb{R}, \\
o_{x}: & =\langle[1,2)+2 \mathbb{Z}\rangle \times \mathbb{R} .
\end{aligned}
$$

Observe that these subsets of $\mathbb{R}^{2}$ satisfy the following relations:

$$
\begin{aligned}
& l \cup r=b \cup t=e_{p} \cup o_{p}=e_{x} \cup o_{x}=\mathbb{R}^{2}, \\
& l \cap r=b \cap t=e_{p} \cap o_{p}=e_{x} \cap o_{x}=\emptyset .
\end{aligned}
$$

\section{The Quantum Propagator}

Our quantization can now be described. We follow the canonical procedure of mapping the function $x$ to the multiplication operator $\widehat{x}$ and the function $p$ to the derivative operator $\widehat{p}=(\hbar / i) d / d x$. We call this mapping $Q_{\hbar}$ and note that it is non-unique due to ordering ambiguities. For the case of the torus, this procedure is used commonly in the mathematics literature (see refs., $, 0,0$ ), but much less so in physics papers. The quantum algebra of observables $\mathfrak{A}_{\hbar}$ is restricted to the set operators generated by the quantization of the classical generators: $Q_{\hbar}(\exp (2 \pi i x))=\exp (2 \pi i \widehat{x})$ and $Q_{\hbar}(\exp (2 \pi i p))=\exp (2 \pi i \widehat{p})$. Following the standard notation, we let

$$
\begin{aligned}
U & =\exp (2 \pi i \widehat{x}) \\
V & =\exp (2 \pi i \widehat{p})
\end{aligned}
$$

so that

$$
U V=e^{4 \pi^{2} i \hbar} V U
$$

We construct a quantum propagator by quantizing the dynamics of the covering map eqn. 2. The quantum dynamics induced on the algebra of observables for the quantum torus is the quantum baker's map.

We now construct the quantum propagator $F$. The kinematics is already given: the Hilbert space is the usual $L^{2}(\mathbb{R})$. For the dynamics, we work in the Heisenberg picture, and first give the quantum analogs of eqns. 1 and 6 . 
We define the following projection operators:

$$
\begin{aligned}
L: & =\int_{[0,1 / 2)+\mathbb{Z}}|x\rangle\langle x| d x, \\
R: & =\int_{[1 / 2,1)+\mathbb{Z}}|x\rangle\langle x| d x, \\
B: & =\int_{[0,1 / 2)+\mathbb{Z}}|p\rangle\langle p| d p, \\
T: & =\int_{[1 / 2,1)+\mathbb{Z}}|p\rangle\langle p| d p,
\end{aligned}
$$

and

$$
\begin{aligned}
E_{x}: & =\int_{[0,1)+2 \mathbb{Z}}|x\rangle\langle x| d x, \\
O_{x}: & =\int_{[1,2)+2 \mathbb{Z}}|x\rangle\langle x| d x, \\
E_{p}: & =\int_{[0,1)+2 \mathbb{Z}}|p\rangle\langle p| d p, \\
O_{p}: & =\int_{[1,2)+2 \mathbb{Z}}|p\rangle\langle p| d p .
\end{aligned}
$$

Observe that

$$
L+R=B+T=E_{x}+O_{x}=E_{p}+O_{p}=I
$$

and

$$
L R=B T=E_{x} O_{x}=E_{p} O_{p}=0 .
$$

We next define appropriate "shift" operators. A shift in $p$, or a shift in $x$, by unity is achieved by the following unitary operators, respectively:

$$
\begin{aligned}
& X=e^{i \widehat{x} / \hbar} \\
& Y=e^{i \hat{p} / \hbar} .
\end{aligned}
$$

Note that $X$ and $Y$ commute with the algebra $\mathfrak{A}_{\hbar}$ generated by $U$ and $V$ :

$$
[X, U]=[Y, U]=[X, V]=[Y, V]=0 .
$$


We shall also need the following commutation relations:

$$
\begin{aligned}
X L & =L X, \quad Y L=L Y, \quad X R=R X, \quad Y R=R Y \\
Y^{1 / 2} L & =R Y^{1 / 2}, \quad X^{1 / 2} B=T X^{1 / 2} \\
Y E_{x} & =O_{x} Y, \quad X E_{p}=O_{p} X .
\end{aligned}
$$

We demonstrate one of these commutation relations explicitly. The others involve similar calculations:

$$
\begin{aligned}
Y^{1 / 2} L & =e^{i \widehat{p} / 2 \hbar} \int_{[0,1 / 2)+\mathbb{Z}}|x\rangle\langle x| d x e^{-i \widehat{p} / 2 \hbar} e^{i \widehat{p} / 2 \hbar} \\
& =\int_{[0,1 / 2)+\mathbb{Z}}|x-1 / 2\rangle\langle x-1 / 2| d x e^{i \widehat{p} / 2 \hbar} \\
& =\int_{[1 / 2,1)+\mathbb{Z}}|x\rangle\langle x| d x e^{i \widehat{p} / 2 \hbar}=R Y^{1 / 2} .
\end{aligned}
$$

We next find the unitary operator $S$ which takes $\widehat{x}$ to $2 \widehat{x}$ and $\widehat{p}$ to $\widehat{p} / 2$. Formally, for any operator expandable as a Taylor series in $\widehat{x} \widehat{p}+\widehat{p} \widehat{x}$, we find

$$
\widehat{x} f(\widehat{x} \widehat{p}+\widehat{p} \widehat{x})=f(\widehat{x} \widehat{p}+\widehat{p} \widehat{x}+2 i \hbar) \widehat{x} .
$$

Thus, if we define the operator

$$
S:=\exp \left(-\frac{i \log 2}{2 \hbar}(\widehat{x} \widehat{p}+\widehat{p} \widehat{x})\right)
$$

we see

$$
\begin{aligned}
\widehat{x} S & =2 S \widehat{x}, \\
\widehat{p} S & =S \widehat{p} / 2,
\end{aligned}
$$

or

$$
\begin{aligned}
S^{\dagger} \widehat{x} S & =2 \widehat{x}, \\
S^{\dagger} \widehat{p} S & =\widehat{p} / 2 .
\end{aligned}
$$

Observe also that the operator $S$ is unitary since $\widehat{x} \widehat{p}+\widehat{p} \widehat{x}$ is Hermitian.

We are now in a position to write down a propagator for the baker's map. Based on eqn. 3, we have the following definition. 
Definition 1 (Baker's Map Propagator) Let the operator $F$ be defined as follows:

$$
\begin{aligned}
F & =S\left(L+X^{-1} R\right)\left(E_{p}+Y^{-1 / 2} O_{p}\right) \\
& =\left(E_{x}+X^{-1 / 2} O_{x}\right)\left(B+Y^{-1} T\right) S
\end{aligned}
$$

Lemma $2 F$ is unitary.

Proof. Observe that $E_{x}=E_{x}^{\dagger}, O_{x}=O_{x}^{\dagger}, B=B^{\dagger}, T=T^{\dagger}$. It follows that

$$
\begin{aligned}
\left(L+X^{-1} R\right)\left(L+X^{-1} R\right)^{\dagger} & =L+R=I, \\
\left(E_{p}+Y^{-1 / 2} O_{p}\right)\left(E_{p}+Y^{-1 / 2} O_{p}\right)^{\dagger} & =E_{p}+O_{p}=I .
\end{aligned}
$$

Thus $F$ is the product of three unitary operators, hence is unitary.

\section{The Classical Limit}

Because of the discontinuity of the baker's map (and its covering map), the classical limit requires more thought (see, for instance, ref. The basic problem comes from the fact that the projection operators $L$ and $E_{p}$ (for example) do not commute as $\hbar \rightarrow 0$ (even weakly). This comes from scaling - each term contributes less, but the number of terms increases.

We demonstrate this explicitly with the following example. Consider a function $\phi \in L^{2}(\mathbb{R})$ which is supported only for $x \in[1 / 2,1)$, and a $\psi$ supported only in $[0,1 / 2)$. Then by construction, we see

$$
\left\langle\psi\left|E_{p} L\right| \phi\right\rangle=0 .
$$

Now consider the action of $L E_{p}$. We see

$$
\begin{aligned}
\left\langle\psi\left|L E_{p}\right| \phi\right\rangle= & \int d x d x^{\prime} \overline{\psi(x)} \phi\left(x^{\prime}\right) \chi_{l}(x)\left\langle x\left|E_{p}\right| x^{\prime}\right\rangle \\
= & \int d x d x^{\prime} \overline{\psi(x)} \phi\left(x^{\prime}\right) \chi_{l}(x)\left\langle x\left|1 / 2-(i / \pi) \sum_{k \text { odd }} \frac{e^{\pi i k \hat{p}}}{k}\right| x^{\prime}\right\rangle \\
= & \frac{1}{2} \int d x d x^{\prime} \chi_{l}(x) \overline{\psi(x)} \phi(x) \\
& -\frac{i}{\pi} \int d x d x^{\prime} \chi_{l}(x) \overline{\psi(x)} \phi\left(x^{\prime}\right)\left\langle x\left|\sum_{k \text { odd }} \frac{e^{\pi i k \widehat{p}}}{k}\right| x^{\prime}\right\rangle .
\end{aligned}
$$


The first term in the expression above is zero, while the second term yields

$$
\begin{aligned}
\left\langle\psi\left|L E_{p}\right| \phi\right\rangle & =-\frac{i}{\pi} \sum_{k \text { odd }} \frac{1}{k} \int d x d x^{\prime} \chi_{l}(x) \overline{\psi(x)} \phi\left(x^{\prime}\right)\left\langle x \mid x^{\prime}-\pi k \hbar\right\rangle \\
& =-\frac{i}{\pi} \sum_{k \text { odd }} \frac{1}{k} \int d x \chi_{l}(x) \overline{\psi(x)} \phi(x+\pi \hbar k) .
\end{aligned}
$$

As a particular example, we choose

$$
\begin{aligned}
& \phi(x)=\chi_{[1 / 2,1)}(x), \\
& \psi(x)=\chi_{[0,1 / 2)}(x) .
\end{aligned}
$$

Then

$$
\begin{aligned}
\left\langle\psi\left|L E_{p}\right| \phi\right\rangle & =-\frac{i}{\pi} \sum_{k \text { odd }} \frac{1}{k} \int_{0}^{1 / 2} d x \chi_{[1 / 2,1)}(x+\pi \hbar k) \\
& =-\frac{i}{\pi} \sum_{k \text { odd }} \frac{1}{k} \int_{0}^{1 / 2} d x \chi_{[1 / 2-\pi \hbar k, 1-\pi \hbar k)}(x)
\end{aligned}
$$

The only terms in the sum not equal to zero have $0 \leq k<\frac{1}{2 \pi \hbar}$ or $\frac{1}{2 \pi \hbar} \leq k<$ $\frac{1}{\pi \hbar}$, so we see

$$
\begin{aligned}
\left\langle\psi\left|L E_{p}\right| \phi\right\rangle & =-\frac{i}{\pi} \sum_{\substack{k \text { odd } \\
\frac{1}{2} \leq \pi \hbar k<1}} \frac{1}{k}(1-\pi \hbar k)-\frac{i}{\pi} \sum_{\substack{k \text { odd } \\
0 \leq \pi \hbar k<1 / 2}} \frac{1}{k}(\pi \hbar k) \\
& \rightarrow-\frac{i}{\pi}\left(\int_{1 / 2 \pi \hbar}^{1 / \pi \hbar}\left(\frac{1}{k}-\pi \hbar\right) d k+\int_{0}^{1 / 2 \pi \hbar} \pi \hbar d k\right) \\
& =-\frac{i}{\pi}((\log 2-1 / 2)+1 / 2) \\
& =-\frac{i \log 2}{\pi} \neq 0 .
\end{aligned}
$$

Thus the limit is finite but nonzero.

All is not lost, however, as we can take a more constrained view of what constitutes a quantum state with a classical limit (see, for example, ref. 10). We take as our quantum state the coherent state $\left|\phi_{\hbar} ; x_{0}, p_{0}\right\rangle$ centered around 
the point $\left(x_{0}, p_{0}\right)$ with a width of $\sqrt{\hbar}$. Recall that a coherent state can be written

$$
\phi_{x_{0}, p_{0}}^{\hbar}(x)=\left\langle x \mid \phi_{\hbar} ; x_{0}, p_{0}\right\rangle=\frac{1}{(\pi \hbar)^{1 / 4}} e^{-\left(x-x_{0}\right)^{2} / 2 \hbar} e^{i p_{0} x / \hbar-i p_{0} x_{0} / 2 \hbar}
$$

with a Fourier transform

$$
\widetilde{\phi}_{x_{0}, p_{0}}^{\hbar}(p)=\left\langle p \mid \phi_{\hbar} ; x_{0}, p_{0}\right\rangle=\frac{1}{(\pi \hbar)^{1 / 4}} e^{-\left(p-p_{0}\right)^{2} / 2 \hbar} e^{-i p x_{0} / \hbar+i p_{0} x_{0} / 2 \hbar} .
$$

We can define the classical limit in terms of these coherent states.

Definition 3 A quantum propagator $F$ is said to have a weak classical limit if for any function on the torus $A$, and for almost every $x_{0}, p_{0}$,

$$
\lim _{\hbar}\left\langle\phi_{\hbar} ; x_{0}, p_{0}\left|F^{\dagger} Q_{\hbar}(A) F\right| \phi_{\hbar} ; x_{0}, p_{0}\right\rangle-\left\langle\phi_{\hbar} ; \beta\left(x_{0}, p_{0}\right)\left|Q_{\hbar}(A)\right| \phi_{\hbar} ; \beta\left(x_{0}, p_{0}\right)\right\rangle
$$

where $\beta$ is the classical evolution.

In other words, if, as $\hbar \rightarrow 0$, any observable has the same value under classical and quantum evolution for almost all wave packets, we say the quantum mechanics yields the classical mechanics. Note also that this differs from the definition given in ref. 10 by use of the "almost all" caveat: we allow the classical limit to fail at a set of measure zero points in phase space.

We then have the following theorem.

Theorem 4 The propagator $F$ defined in eqn. 11 has a weak classical limit in the sense of definition 3.

Proof. We give the proof for a harmonic $Q_{\hbar}(A)=U^{a} V^{b}$. The general case will follow by linearity and continuity. We divide the proof into steps.

Step 1. We first calculate the expectation value of the operator $U^{a} V^{b}$ in the coherent states. We see

$$
\begin{aligned}
& \left\langle\phi_{\hbar} ; x_{0}, p_{0}\left|U^{a} V^{b}\right| \phi_{\hbar} ; x_{0}, p_{0}\right\rangle \\
= & \frac{1}{(\pi \hbar)^{1 / 2}} \int d x d x^{\prime} e^{-\left(x-x_{0}\right)^{2} / 2 \hbar} e^{-i p_{0} x / \hbar} e^{2 \pi i a x} \delta\left(x-x^{\prime}-2 \pi b \hbar\right) e^{-\left(x^{\prime}-x_{0}\right)^{2} / 2 \hbar} e^{i p_{0} x^{\prime} / \hbar} \\
= & e^{2 \pi i b p_{0}} e^{2 \pi i a x_{0}} e^{-2 \pi^{2} b^{2} \hbar} e^{-4 \pi^{2} \hbar(b+i a)^{2}} \rightarrow e^{2 \pi i b p_{0}} e^{2 \pi i a x_{0}} \quad \text { as } \hbar \rightarrow 0 .
\end{aligned}
$$


Step 2. Observe that acting on these states, we see

$$
\begin{aligned}
\| L\left|\phi_{\hbar} ; x_{0}, p_{0}\right\rangle \|^{2} & =\left\langle\phi_{\hbar} ; x_{0}, p_{0}|L| \phi_{\hbar} ; x_{0}, p_{0}\right\rangle \\
& =\frac{1}{(\pi \hbar)^{1 / 2}} \sum_{k \in \mathbb{Z}} \int_{0}^{1 / 2} e^{-\left(x+k-x_{0}\right)^{2} / \hbar} d x .
\end{aligned}
$$

Suppose $x_{0} \neq l / 2$ for $l \in \mathbb{Z}$. Now choose $\epsilon>0$ and $l \in \mathbb{Z}$ such that $x_{0} \in[l / 2+\epsilon,(l+1) / 2-\epsilon)$ for $\epsilon>0$.

For the case of $l$ odd, we see that the value of the integral is bounded by

$$
\left|\frac{1}{(\pi \hbar)^{1 / 2}} \sum_{k \in \mathbb{Z}} \int_{0}^{1 / 2} e^{-\left(x-x_{0}+k\right)^{2} / \hbar} d x\right| \leq \frac{2}{\sqrt{\pi}} \int_{\epsilon / \sqrt{\hbar}}^{\infty} e^{-x^{2}} d x .
$$

A bound on this integral can easily be given for $\epsilon / \sqrt{\hbar}>1$. We see that

$$
\int_{\epsilon / \sqrt{\hbar}}^{\infty} e^{-x^{2}} d x \leq \int_{\epsilon / \sqrt{\hbar}}^{\infty} x e^{-x^{2}} d x=\frac{1}{2} \int_{\epsilon / \sqrt{\hbar}}^{\infty} e^{-u} d u=e^{-\epsilon^{2} / \hbar} / 2 .
$$

Thus for $l$ odd, the limit of the integral is zero as $\hbar \rightarrow 0$.

Now consider $x_{0} \in[l / 2+\epsilon,(l+1) / 2-\epsilon)$ with $l$ even. Then we see that

$$
\begin{aligned}
\| L\left|\phi_{\hbar} ; x_{0}, p_{0}\right\rangle \|^{2} & =\|(I-R)\left|\phi_{\hbar} ; x_{0}, p_{0}\right\rangle \|^{2} \\
& =1-\| R\left|\phi_{\hbar} ; x_{0}, p_{0}, \mu\right\rangle \|^{2} \\
& \rightarrow 1 \text { as } \hbar \rightarrow 0 .
\end{aligned}
$$

Similar results hold for all the projection operators $L, R, B, T, E_{x}, O_{x}, E_{p}, O_{p}$ defined in eqns. 7 and 8 . We let $\tilde{l}, \tilde{r}, \tilde{b}, \tilde{t}, \tilde{e}_{x}, \tilde{o}_{x}, \tilde{e}_{p}, \tilde{o}_{p}$ denote the interior of the regions given in eqns. 1 and 6, that is the regions with the boundaries removed. (For instance $\widetilde{l}$ does not contain $x=0$ or $x=1 / 2$.) Note that $\left(\widetilde{l} \cap \widetilde{e}_{p}\right) \cup\left(\widetilde{r} \cap \widetilde{e}_{p}\right) \cup\left(\widetilde{l} \cap \widetilde{o}_{p}\right) \cup\left(\widetilde{r} \cap \widetilde{o}_{p}\right)$ is dense in $\mathbb{R}^{2}$. We summarize these results in Table 1.

Step 3. Now suppose $\left(x_{0}, p_{0}\right) \in \tilde{r} \cap \widetilde{o}_{p}$. Then consider the quantum evolution. We see

$$
\begin{aligned}
& \left\langle\phi_{\hbar} ; x_{0}, p_{0}\left|\left(E_{p}+Y^{1 / 2} O_{p}\right)(L+X R) S^{\dagger} U^{a} V^{b} S\left(L+X^{-1} R\right)\left(E_{p}+Y^{-1 / 2} O_{p}\right)\right| \phi_{\hbar} ; x_{0}, p_{0}\right\rangle \\
= & \left\langle\phi_{\hbar} ; x_{0}, p_{0}\left|\left(E_{p}+Y^{1 / 2} O_{p}\right)(L+X R) U^{2 a} V^{b / 2}\left(L+X^{-1} R\right)\left(E_{p}+Y^{-1 / 2} O_{p}\right)\right| \phi_{\hbar} ; x_{0}, p_{0}\right\rangle
\end{aligned}
$$


Multiplying out, we see 16 terms in the expansion. Consider one of these terms. We see from Table 1 that

$$
\begin{aligned}
& \left|\left\langle\phi_{\hbar} ; x_{0}, p_{0}\left|E_{p} X R U^{2 a} V^{b / 2} L Y^{-1 / 2} O_{p}\right| \phi_{\hbar} ; x_{0}, p_{0}\right\rangle\right| \\
= & \left|\left\langle\phi_{\hbar} ; x_{0}, p_{0}\left|E_{p} R U^{2 a+N} V^{(b-N) / 2} R O_{p}\right| \phi_{\hbar} ; x_{0}, p_{0}\right\rangle\right| \\
\leq & \left|\left\langle\phi_{\hbar} ; x_{0}, p_{0}\left|E_{p}\right| \phi_{\hbar} ; x_{0}, p_{0}\right\rangle\right| \\
& \times\left|\left\langle\phi_{\hbar} ; x_{0}, p_{0}\left|\left(R U^{2 a+N} V^{(b-N) / 2} R O_{p}\right)^{\dagger}\left(R U^{2 a+N} V^{(b-N) / 2} R O_{p}\right)\right| \phi_{\hbar} ; x_{0}, p_{0}\right\rangle\right| \\
\rightarrow & 0 \text { as } \hbar \rightarrow 0 \text { since }\left(x_{0}, p_{0}\right) \in \widetilde{o}_{p} .
\end{aligned}
$$

Note in the second step we have used the Schwartz inequality and the identity $E_{p}^{2}=E_{p}$. Similarly, 15 of the terms vanish as $\hbar \rightarrow 0$. The only surviving term for $\left(x_{0}, p_{0}\right) \in \tilde{r} \cap \widetilde{o}_{p}$ is

$$
\begin{aligned}
& \left\langle\phi_{\hbar} ; x_{0}, p_{0}\left|O_{p} Y^{1 / 2} L U^{2 a} V^{b / 2} L Y^{-1 / 2} O_{p}\right| \phi_{\hbar} ; x_{0}, p_{0}\right\rangle \\
\rightarrow & \left\langle\phi_{\hbar} ; x_{0}, p_{0}\left|U^{2 a} V^{b / 2}\right| \phi_{\hbar} ; x_{0}, p_{0}\right\rangle \\
\rightarrow & e^{2 \pi i\left(2 a x_{0}+(b / 2) p_{0}\right)} .
\end{aligned}
$$

Step 4. Now consider the classical evolution. We have, for $\left(x_{0}, p_{0}\right) \in \tilde{r} \cap \widetilde{o}_{p}$

$$
\begin{aligned}
\left\langle\phi_{\hbar} ; \beta\left(x_{0}, p_{0}\right)\left|U^{a} V^{b}\right| \phi_{\hbar} ; \beta\left(x_{0}, p_{0}\right)\right\rangle & =\left\langle\phi_{\hbar} ; 2 x_{0}, p_{0} / 2\left|U^{a} V^{b}\right| \phi_{\hbar} ; 2 x_{0}, p_{0} / 2\right\rangle \\
& \rightarrow e^{2 \pi i\left(2 a x_{0}+(b / 2) p_{0}\right)} .
\end{aligned}
$$

The calculations for the other regions are similar, and we omit the details here. This concludes the proof.

\section{Planck's Constant $=1 / N$}

A remarkable set of properties can be associated with quantum dynamics on the torus if we let Planck's constant satisfy the integrality condition

$$
h=1 / N
$$

This fact is evidenced by the quantization schemes presented in ref. 1 and ref 2 for the baker's map. In ref.5 a procedure was developed to obtain a finite-dimensional quantum cat-dynamics for the case of periodic boundary conditions, and it was found to have the same form as the original matrix quantization proposed in ref..11 
We shall find for the baker's map, a finite-dimensional matrix propagator for $N$ even given by

$$
\left(\Phi_{n}^{(0,0)}, F \Phi_{m}^{(0,0)}\right)_{P}=\left[(\mathcal{Z})\left(\mathcal{F}^{N}\right)^{-1}\left(\begin{array}{ll}
\mathcal{F}^{N / 2} & 0 \\
0 & -\mathcal{F}^{N / 2}
\end{array}\right)\left(\mathcal{Z}^{-2}\right)\right]_{n m}
$$

where the indices $n$ and $m$ take values between 0 and $N-1$ and we use the following notations: (1) $\mathcal{F}^{N}$ is the $N \times N$ discrete Fourier transform matrix defined in eqn. 21 below; (2) $\mathcal{Z}$ is the diagonal matrix

$$
(\mathcal{Z})_{n m}=\delta_{n, m} e^{i \pi n / N}
$$

(3) $\Phi_{n}^{(0,0)}$ is a basis vector of the Hilbert space $\mathcal{H}_{\hbar}(0) \cong \mathbb{C}^{N}$ defined as the periodic $\delta$-comb (eqn. 18); and $(4)(\cdot, \cdot)_{P}$ is the inner product defined in eqn. 17 .

Since $\Phi_{n+N}^{(0,0)}=\Phi_{n}^{(0,0)}$, the extended matrix for general $n$ and $m \in \mathbb{Z}$ is periodic with period $N$. Thus the form of eqn. 14 may be misleading for $n, m$ outside of the fundamental range $[0, N-1]$. In general, we write $(\mathcal{Z})_{n m}=\delta_{n, m} e^{i \pi(n / N-[n / N])}$ where $[n / N]$ represents the integer part of $n / N$.

In ref., 1 the following matrix propagator was proposed by Balazs and Voros:

$$
\sum_{a=0}^{N / 2-1}\left(\mathcal{F}^{N}\right)_{n a}^{-1}\left(\begin{array}{ll}
\mathcal{F}^{N / 2} & 0 \\
0 & \mathcal{F}^{N / 2}
\end{array}\right)_{a m}
$$

It can be easily verified that for $n$ even, the results are identical, while for $n$ odd, this differs from eqn. 13 by a phase $e^{i \pi \zeta / N}$ where $\zeta=n-2 m$ for $0 \leq m<N / 2$ or $\zeta=n-2(m-N / 2)$ for $N / 2 \leq m<N$. These phases become small along classical trajectories.

Indeed, in the small $\hbar$ limit we can see that these phases are $O(\hbar)$ corrections. Consider, for example the case $0 \leq m<N / 2$. In, 1 it was shown that the matrix elements (eqn. 15) for $n$ odd can be written as

$$
\frac{\sqrt{2}}{N}\left(1+i \cot \frac{\pi}{N}(n-2 m)\right) \text {. }
$$

We let $n=N \chi$ and $m=N \xi$ with $\chi, \xi \in[0,1 / 2]$ to see the behavior as $N \rightarrow \infty$. Then, the above expression behaves like

$$
\frac{\sqrt{2}}{N}(1+i \cot \pi(\chi-2 \xi)) \rightarrow 0
$$


unless $\chi-2 \xi=O(1 / N)=O(\hbar)$. Thus the phase change is $O(\hbar)$ for the non-vanishing matrix elements.

Furthermore, the matrix propagator (eqn. 13) is shown below to preserve the symmetry $x \rightarrow 1-x$ and $p \rightarrow 1-p$, which is not preserved in the original quantization given by Balazs and Voros.t (In Saraceno, 2 an antiperiodic quantization is formulated which does preserve this symmetry.) In addition, we verify that this new matrix propagator satisfies the classical symmetry $x \rightarrow p, p \rightarrow x$, and $t \rightarrow-t$.

\section{A. The $\theta$-torus}

For $h=1 / N$ the algebra generated by $U$ and $V$ has a natural center generated by

$$
\begin{aligned}
& X=U^{N}, \\
& Y=V^{N}
\end{aligned}
$$

That is

$$
[X, Y]=[X, U]=[X, V]=[Y, U]=[Y, V]=0 .
$$

In ref.5 and, 6 this insight was used to show that $L^{2}(\mathbb{R})$ can be decomposed via the following eigenvalue problem:

$$
\begin{aligned}
& X \Phi=e^{2 \pi i \theta_{1}} \Phi \\
& Y \Phi=e^{2 \pi i \theta_{2}} \Phi,
\end{aligned}
$$

where $\theta=\left(\theta_{1}, \theta_{2}\right) \in \mathbb{T}^{2}$. As in ref. 自 let $\mathcal{H}_{\hbar}(\theta)$ denote the space of (nonnormalizable) independent eigenvectors with fixed $\theta$. The space $\mathcal{H}_{\hbar}(\theta)$ has a natural inner product defined as an integral over the fundamental domain $D=[0,1] \subset \mathbb{R}$ given by

$$
\left(\Psi_{1}(\theta), \Psi_{2}(\theta)\right)_{P}=\int_{0}^{1} \overline{\Psi_{1}(x, \theta)}\left(K \Psi_{2}\right)(x, \theta) d x,
$$

where

$$
\begin{aligned}
K \Psi_{2}(x, \theta) & =\int_{-\infty}^{\infty} K(x, y) \Psi_{2}(y, \theta) d y \\
K(x, y) & =\frac{1}{2 \pi \hbar} g\left(\frac{x-y}{2 \hbar}\right)
\end{aligned}
$$


and

$$
g(r)=\frac{\sin r}{r} e^{-\hbar r^{2}+i r} .
$$

The following lemma was proved in 6 :

Lemma 5 (i) The following (generalized) functions are elements of $\mathcal{H}_{\hbar}(\theta)$ of unit norm:

$$
\Phi_{m}^{(\theta)}(x)=\frac{e^{2 \pi i \theta_{2} m / N}}{N^{1 / 2}} \sum_{k \in \mathbb{Z}} e^{2 \pi i \theta_{2} k} \delta\left(x-\frac{m+\theta_{1}+N K}{N}\right) .
$$

They are periodic in $m$,

$$
\Phi_{m+N}^{(\theta)}=\Phi_{m}^{(\theta)}
$$

and furthermore,

$$
\Phi_{0}^{(\theta)}, \ldots, \Phi_{N-1}^{(\theta)}
$$

are orthogonal vectors in $\mathcal{H}_{\hbar}(\theta)$.

(ii) The space $\mathcal{H}_{\hbar}(\theta)$ has dimension $N$. Consequently the functions 18 form an orthonormal basis for $\mathcal{H}_{\hbar}(\theta)$.

We use the following notation for these vectors:

$$
\Phi_{m}^{(\theta)}=\frac{e^{2 \pi i \theta_{2} m / N}}{N^{1 / 2}} \sum_{k \in \mathbb{Z}} e^{2 \pi i \theta_{2} k}\left|\frac{\theta_{1}+m}{N}+k\right\rangle_{x}
$$

These are the $\delta$-comb wavefunctions seen for example in ref.11 We can, of course, just as easily work in momentum representation.

Lemma 6 For $h=1 / N$

$$
\Phi_{m}^{(\theta)}=e^{-2 \pi i \theta_{1} \theta_{2} / N} \sum_{n=0}^{N-1} \mathcal{F}_{m n}^{N} \widetilde{\Phi}_{n}^{(\theta)},
$$

where $\left\{\widetilde{\Phi}_{n}^{(\theta)}\right\}_{0 \leq n \leq N-1}$ are the momentum-state wave functions on the torus,

$$
\widetilde{\Phi}_{n}^{(\theta)}=\frac{e^{-2 \pi i n \theta_{1} / N}}{\sqrt{N}} \sum_{k} e^{-2 \pi i \theta_{1} k}\left|\frac{\theta_{2}+n}{N}+k\right\rangle_{p},
$$


and $\mathcal{F}_{m n}^{N}$ is the matrix for the discrete Fourier transform,

$$
\mathcal{F}_{m n}^{N}=\frac{e^{-2 \pi i m n / N}}{\sqrt{N}}
$$

In particular, for the subsets $\theta_{1}=0$ or $\theta_{2}=0$, changing coordinates from momentum representation to position representation is simply a discrete Fourier transform.

\section{Dynamics at $\theta=(0,0)$}

The point $\theta=(0,0)$ of the $\theta$-torus corresponds to the $N$-dimensional vector space $\mathcal{H}_{\hbar}(0)$ of periodic $\delta$-combs. For the quantum baker's map, we now show that $\theta=(0,0)$ is an invariant point of the dynamics on the $\theta$-torus for $N$ even. That is, the set of periodic $\delta$-combs is mapped onto itself by our propagator $F$. We have, using the commutation relations eqn. 9,

$$
X F \Phi_{m}^{(0,0)}=F X^{2} \Phi_{m}^{(0,0)}=F \Phi_{m}^{(0,0)}
$$

and

$$
\begin{aligned}
Y F \Phi_{m}^{(0,0)} & =\left(O_{x}+X^{-1 / 2} E_{x}\right)\left(B+Y^{-1} T\right) S Y^{1 / 2} \Phi_{m}^{(0,0)} \\
& =\left(X^{1 / 2} O_{x}+E_{x}\right)\left(T+Y^{-1} B\right) S X^{-1} Y^{1 / 2} \Phi_{m}^{(0,0)} \\
& =\left(E_{x}+X^{1 / 2} O_{x}\right)(B+Y T) S X^{-1} Y \Phi_{m}^{(0,0)} \\
& =E_{x}(B+Y T) S X^{-1} Y \Phi_{m}^{(0,0)}+X^{-1 / 2} O_{x}(B+Y T) S X Y \Phi_{m}^{(0,0)} \\
& =E_{x}\left(B+Y^{-1} T\right) S \Phi_{m}^{(0,0)}+X^{-1 / 2} O_{x}\left(B+Y^{-1} T\right) S \Phi_{m}^{(0,0)} \\
& =F \Phi_{m}^{(0,0)}
\end{aligned}
$$

Observe that we can write any $A$ as $\sum_{j, k} \gamma_{j k} U^{j} V^{k}$. Letting $j=a+N c$ and $k=b+N d$ with $0 \leq a, b \leq N-1$ and $c, d \in \mathbb{Z}$, we see from eqn. 16 that

$$
U^{a+N c} V^{b+N d} \Phi_{m}^{(0,0)}=U^{a} V^{b} X^{c} Y^{d} \Phi_{m}^{(0,0)}=U^{a} V^{b} \Phi_{m}^{(0,0)} .
$$

Thus, acting on the subspace $\mathcal{H}(0)$, the algebra $\mathfrak{A}_{\hbar}$ is reduced to a set of $N^{2}$ operators. This is isomorphic to the algebra of $N \times N$ matrices. One of these operators is the propagator $F$, and it remains to determine the matrix elements. 
Theorem 7 The matrix elements for the propagator $F$ on the subspace $\mathcal{H}(0)$ are given by eqn. 1 .

Proof. We divide this calculation into different cases. For $0 \leq m<N / 2$, we have

$$
\begin{aligned}
& F \Phi_{m}^{(0,0)}=\left(E_{x}+X^{-1 / 2} O_{x}\right)\left(B+Y^{-1} T\right) S \Phi_{m}^{(0,0)} \\
& =\left(E_{x}+X^{-1 / 2} O_{x}\right)\left(B+Y^{-1} T\right) \sqrt{\frac{2}{N}} \sum_{k \in \mathbb{Z}}\left|\frac{2 m}{N}+2 k\right\rangle \\
& =\frac{1}{\sqrt{2}}\left(E_{x}+X^{-1 / 2} O_{x}\right)\left(B+Y^{-1} T\right)\left(\Phi_{2 m}^{(0,0)}+e^{-2 \pi i m / N} \Phi_{2 m}^{(0,1 / 2)}\right) \\
& =\frac{1}{\sqrt{2}} \Phi_{2 m}^{(0,0)}+\frac{e^{-2 \pi i m / N}}{\sqrt{2}}\left(E_{x}+X^{-1 / 2} O_{x}\right)(B-T) \Phi_{2 m}^{(0,1 / 2)} .
\end{aligned}
$$

Now, observe that

$$
\begin{aligned}
B \Phi_{m}^{(0,1 / 2)} & =\frac{e^{i \pi m / N}}{\sqrt{N}} \sum_{k \in \mathbb{Z}}(-1)^{k} \int_{[0,1 / 2)+\mathbb{Z}}|p\rangle\left\langle p \mid \frac{m}{N}+k\right\rangle_{x} d p \\
& =e^{i \pi m / N} \int_{[0,1 / 2)+\mathbb{Z}}|p\rangle\left(\sum_{k \in \mathbb{Z}} e^{2 \pi i k(1 / 2-N p)}\right) e^{-2 \pi i p m} d p \\
& =\frac{e^{i \pi m / N}}{\sqrt{N}} \sum_{a=0}^{N / 2-1} e^{-2 \pi i(a+1 / 2) m / N} \frac{1}{\sqrt{N}} \sum_{k \in \mathbb{Z}}\left|\frac{a+1 / 2}{N}+k\right\rangle_{p} \\
& =\frac{1}{\sqrt{N}} \sum_{a=0}^{N / 2-1} e^{-2 \pi i a m / N} \widetilde{\Phi}_{a}^{(0,1 / 2)}
\end{aligned}
$$

where we have used the "p-state" $\delta$-comb given in 20. Thus, we see that

$(B-T) \Phi_{m}^{(0,1 / 2)}=\frac{1}{\sqrt{N}}\left(\sum_{a=0}^{N / 2-1} e^{-2 \pi i a m / N}-\sum_{a=N / 2}^{N-1} e^{-2 \pi i a m / N}\right) \sum_{b=0}^{N-1}\left(\mathcal{F}^{-1}\right)_{a b} \Phi_{b}^{(0,1 / 2)}$

and

$$
F \Phi_{m}^{(0,0)}=\frac{1}{\sqrt{2}} \Phi_{2 m}^{(0,0)}
$$




$$
\begin{aligned}
& +\frac{e^{-2 \pi i m / N}}{\sqrt{2 N}}\left(\sum_{a=0}^{N / 2-1} e^{-2 \pi i a(2 m) / N}-\sum_{a=N / 2}^{N-1} e^{-2 \pi i a(2 m) / N}\right) \\
& \times \sum_{b=0}^{N-1}\left(\mathcal{F}^{-1}\right)_{a b}\left(E_{x}+(-1)^{b} O_{x}\right) \Phi_{b}^{(0,1 / 2)} .
\end{aligned}
$$

Now oberve

$$
E_{x} \Phi_{m}^{(0,1 / 2)}=\frac{e^{i \pi m / N}}{\sqrt{N}} \sum_{k \in \mathbb{Z}} e^{i \pi k} \chi_{e_{x}}\left(\frac{m}{N}+k\right)\left|\frac{m}{N}+k\right\rangle_{x} .
$$

So for $m \in[1, N-1]$, this yields

$$
\begin{aligned}
E_{x} \Phi_{m}^{(0,1 / 2)} & =\frac{e^{i \pi m / N}}{\sqrt{N}} \sum_{k \in \text { even }} e^{i \pi k}\left|\frac{m}{N}+k\right\rangle_{x}=\frac{e^{i \pi m / N}}{\sqrt{N}} \sum_{k \in \mathbb{Z}}\left|\frac{m}{N}+2 k\right\rangle_{x} \\
& =\frac{\Phi_{m}^{(0,1 / 2)}+e^{i \pi m / N} \Phi_{m}^{(0,0)}}{2} .
\end{aligned}
$$

We let $[m / N]$ be the integer part of $m / N$. Then

$$
\begin{aligned}
E_{x} \Phi_{m}^{(0,1 / 2)} & =\frac{1}{2}\left(\Phi_{m}^{(0,1 / 2)}+e^{i \pi(m / N-[m / N])} \Phi_{m}^{(0,0)}\right) \\
O_{x} \Phi_{m}^{(0,1 / 2)} & =\frac{1}{2}\left(\Phi_{m}^{(0,1 / 2)}-e^{i \pi(m / N-[m / N])} \Phi_{m}^{(0,0)}\right)
\end{aligned}
$$

and hence

$$
\left(E_{x}-O_{x}\right) \Phi_{m}^{(0,1 / 2)}=e^{i \pi(m / N-[m / N])} \Phi_{m}^{(0,0)} .
$$

(Note that we have avoided the case $m=0$ since eqn. 22 has only odd terms in the sum. There is a subtelty involved in this case coming from the fact that $\Phi_{m}^{(0,0)}$ occurs on the boundary.)

Thus,

$$
\begin{aligned}
F \Phi_{m}^{(0,0)}= & \frac{1}{\sqrt{2}} \Phi_{2 m}^{(0,0)} \\
& +\frac{e^{-2 \pi i m / N}}{\sqrt{2 N}}\left(\sum_{a=0}^{N / 2-1} e^{-2 \pi i a(2 m) / N}-\sum_{a=N / 2}^{N-1} e^{-2 \pi i a(2 m) / N}\right) \sum_{b \text { even }}^{N-1}\left(\mathcal{F}^{-1}\right)_{a b} \Phi_{b}^{(0,1 / 2)} \\
& +\frac{e^{-2 \pi i m / N}}{\sqrt{2 N}}\left(\sum_{a=0}^{N / 2-1} e^{-2 \pi i a(2 m) / N}-\sum_{a=N / 2}^{N-1} e^{-2 \pi i a(2 m) / N}\right) \sum_{b \text { odd }}^{N-1} e^{i \pi b / N}\left(\mathcal{F}^{-1}\right)_{a b} \Phi_{b}^{(0,0)} .
\end{aligned}
$$


Consider just the middle term. Since we have already shown that $\theta=(0,0)$ is a fixed point of the dynamics, we should see this term exactly vanishing. In fact a direct calculation readily shows this. For $b$ even

$$
\begin{aligned}
& \frac{e^{-2 \pi i m / N}}{\sqrt{2 N}}\left(\sum_{a=0}^{N / 2-1} e^{-2 \pi i a(2 m) / N}-\sum_{a=N / 2}^{N-1} e^{-2 \pi i a(2 m) / N}\right)\left(\mathcal{F}^{-1}\right)_{a b} \\
= & \frac{e^{-2 \pi i m / N}}{N \sqrt{2}}\left(\sum_{a=0}^{N / 2-1} e^{-2 \pi i a(2 m) / N} e^{2 \pi i a b / N}-\sum_{a=0}^{N / 2-1} e^{-2 \pi i(a+N / 2)(2 m) / N} e^{2 \pi i(a+N / 2) b / N}\right) \\
= & 0 .
\end{aligned}
$$

Thus,

$$
\begin{aligned}
F \Phi_{m}^{(0,0)}= & \frac{1}{\sqrt{2}} \Phi_{2 m}^{(0,0)} \\
& +\frac{e^{-2 \pi i m / N}}{\sqrt{2 N}}\left(\sum_{a=0}^{N / 2-1} e^{-2 \pi i a(2 m) / N}-\sum_{a=N / 2}^{N-1} e^{-2 \pi i a(2 m) / N}\right) \sum_{b \text { odd }}^{N-1} e^{i \pi b / N}\left(\mathcal{F}^{-1}\right)_{a b} \Phi_{b}^{(0,0)} .
\end{aligned}
$$

We next calculate the matrix elements. We see

$$
\left(\Phi_{n}^{(0,0)}, F \Phi_{m}^{(0,0)}\right)_{P}= \begin{cases}\sum_{a=0}^{N / 2-1}\left(\mathcal{F}^{N}\right)_{n a}^{-1}\left(\begin{array}{ll}
\mathcal{F}^{N / 2} & 0 \\
0 & 0
\end{array}\right)_{a m}^{a m} & n \text { even } \\
e^{i \pi(n-2 m) / N} \sum_{a=0}^{N / 2-1}\left(\mathcal{F}^{N}\right)_{n a}^{-1}\left(\begin{array}{ll}
\mathcal{F}^{N / 2} & 0 \\
0 & 0
\end{array}\right)_{a m} n \text { odd }\end{cases}
$$

For the case $N / 2 \leq m<N$, we see

$$
\begin{aligned}
F \Phi_{m}^{(0,0)}= & \left(E_{x}+X^{-1 / 2} O_{x}\right)\left(B+Y^{-1} T\right) \sqrt{\frac{2}{N}} \sum_{k \in \mathbb{Z}}\left|\frac{2 m-N}{N}+2 k+1\right\rangle \\
= & \frac{1}{\sqrt{2}} \phi_{2 m-N}^{(0,0)}-\frac{e^{-2 \pi i m / N}}{\sqrt{2 N}}\left(\sum_{a=N / 2}^{N-1} e^{-2 \pi i a(2 m) / N}-\sum_{a=N / 2}^{N-1} e^{-2 \pi i a(2 m) / N}\right) \\
& \times \sum_{b \text { odd }}^{N-1} e^{i \pi b / N}\left(\mathcal{F}^{-1}\right)_{a b} \Phi_{b}^{(0,1 / 2)}
\end{aligned}
$$


Thus,

$$
\left(\Phi_{n}^{(0,0)}, F \Phi_{m}^{(0,0)}\right)_{P}= \begin{cases}\sum_{a=0}^{N / 2-1}\left(\mathcal{F}^{N}\right)_{n a}^{-1}\left(\begin{array}{ll}
0 & 0 \\
0 & \mathcal{F}^{N / 2}
\end{array}\right)_{a m} & n \text { even } \\
-e^{i \pi(n-2 m) / N} \sum_{a=0}^{N / 2-1}\left(\mathcal{F}^{N}\right)_{n a}^{-1}\left(\begin{array}{ll}
0 & 0 \\
0 & \mathcal{F}^{N / 2}
\end{array}\right)_{a m} n \text { odd }\end{cases}
$$

Combining these results, we see that eqn. 13 holds for any $m$ and $n$. This completes the proof of the theorem.

It is easy to see that the matrix $(B)_{n m}=\left(\Phi_{n}^{(0,0)}, F \Phi_{m}^{(0,0)}\right)$ is unitary, since from eqn. 13 it is the product of four unitary $N \times N$ matrices.

\section{Symmetries in the Quantum Dynamics}

We conclude with a demonstration that the quantization prescription we use preserves the two classical symmetries at $\theta=(0,0)$ : (1) Parity, and (2) Time-Reversal.

(1) Parity

One of the benfits of the quantization prescription given above is the that the classical parity operation $x \rightarrow 1-x, p \rightarrow 1-p$ is preserved at the point $\theta=(0,0)$ in the quantum dynamics. Let us discuss the classical symmetry first. A map $\beta:(x, p) \rightarrow\left(x^{\prime}, p^{\prime}\right)$ is said to be symmetric under the operation $s:(x, p) \rightarrow(\widetilde{x}, \widetilde{p})$ if $\beta^{*} s^{*} f=s^{*} \beta^{*} f$, where the $*$ denotes the pullback of the map on the classical algebra of observables (here the periodic functions). It is easy to see that this property holds classically. Acting on a harmonic $e^{2 \pi i(a x+b p)}$, we see

$$
\begin{aligned}
s^{*} \beta^{*} e^{2 \pi i(a x+b p)} & =-e^{-4 \pi i a x} e^{-i \pi b p}\left(\chi_{r}(x)+(-1)^{b} \chi_{l}(x)\right)\left(\chi_{o_{p}}(p)+(-1)^{b} \chi_{e_{p}}(p)\right) \\
& =-e^{-4 \pi i a x} e^{-i \pi b p}\left(\chi_{l}(x)+(-1)^{b} \chi_{r}(x)\right)\left(\chi_{e_{p}}(p)+(-1)^{b} \chi_{o_{p}}(p)\right) \\
& =\beta^{*} s^{*} e^{2 \pi i(a x+b p)}
\end{aligned}
$$

The case of a more general function follows readily from linearity and continuity.

In the quantum dynamics, we look only at the case $\theta=(0,0)$ and we define the operation of conjugation as

$$
\begin{aligned}
& P|x\rangle=|-x\rangle, \\
& P|p\rangle=|-p\rangle
\end{aligned}
$$


Observe that $P=P^{\dagger}=P^{-1}$ and

$$
P U^{a} V^{b} P=U^{-a} V^{-b}
$$

In fact it is easy to see that on the Hilbert space $\mathcal{H}_{\hbar}(0)$ is invariant under $P$. Explicitly,

$$
P \Phi_{m}^{(0,0)}=\Phi_{N-m}^{(0,0)}
$$

and

$$
\begin{aligned}
P \widetilde{\Phi}_{n}^{(0,0)} & =\frac{1}{\sqrt{N}} \sum_{m=0}^{N-1} e^{-2 \pi i n m / N} P \Phi_{m}^{(0,0)} \\
& =\frac{1}{\sqrt{N}} \sum_{m=0}^{N-1} e^{-2 \pi i n m / N} \Phi_{N-m}^{(0,0)} \\
& =\frac{1}{\sqrt{N}} \sum_{m^{\prime}=0}^{N-1} e^{-2 \pi i(N-n) m / N} \Phi_{m}^{(0,0)} \\
& =\widetilde{\Phi}_{N-n}^{(0,0)} .
\end{aligned}
$$

It is now straightforward to check that $P F P=F$ on $\Phi_{m}^{(0,0)}$. Consider first the case $n>0$, even. Then

$$
\begin{aligned}
(P F P)_{n m} & =\frac{1}{\sqrt{2}}\left(\delta_{N-n, 2 N-2 m}+\delta_{N-n, N-2 m}\right) \\
& =\frac{1}{\sqrt{2}}\left(\delta_{n, 2 m}+\delta_{n, 2 m-N}\right)=F_{n m}
\end{aligned}
$$

At $n=0$,

$$
F_{0 m}=\frac{1}{\sqrt{2}}\left(\delta_{0, m}+\delta_{N / 2, m}\right)
$$

but $P$ is simply the identity operator on $\Phi_{0}^{(0,0)}$ and $\Phi_{N / 2}^{(0,0)}$.

For the case $n$ odd, we simply multiply the Balazs-Voros matrix elements by the extra phase $\exp (i \pi((n-2 m) / N-[2 m / N]))$ to get

$$
F_{n m}=\frac{e^{i \pi(n-2 m) / N}}{N \sqrt{2}}(1+i \cot (\pi(n-2 m) / N)) .
$$


Thus

$$
\begin{aligned}
(P F P)_{n m} & =-\frac{e^{i \pi(2 m-n) / N}}{N \sqrt{2}}(1+i \cot (\pi(2 m-n) / N)) \\
& =-\frac{e^{i \pi(2 m-n) / N}}{N \sqrt{2}}\left(1-\frac{e^{i \pi(2 m-n) / N}+e^{-i \pi(2 m-n) / N}}{e^{i \pi(2 m-n) / N}-e^{-i \pi(2 m-n) / N}}\right) \\
& =-\frac{e^{i \pi(2 m-n) / N}}{N \sqrt{2}}\left(\frac{-2 e^{-i \pi(2 m-n) / N}}{e^{i \pi(2 m-n) / N}-e^{-i \pi(2 m-n) / N}}\right) \\
& =F_{n m} .
\end{aligned}
$$

Thus on the subspace $\mathcal{H}_{\hbar}(0)$ parity is conserved. Observe that the matrix propagator we get is slightly different from that given in. We see now that the extra phases (which vanish as $\hbar \rightarrow 0$ ) are precisely the terms necessary to return this classical symmetry in the quantum dynamics.

(2) Time-Reversal

The classical baker's map also exhibits a time-reversal symmetry under the following transformation:

$$
\begin{aligned}
& x \rightarrow p, \\
& p \rightarrow x, \\
& t \rightarrow-t .
\end{aligned}
$$

The quantization given in ref exhibits this symmetry, and, as we shall now verify, the quantum propagator we have given here does as well. We define an anti-linear operator $\Omega$ which takes $x$ to $p$ through its action on a position eigenstate $|a\rangle_{x}$ :

$$
\Omega|a\rangle_{x}=|a\rangle_{p}
$$

In other words, $\Omega$ transforms a state at position $a$ to a state with momentum a. We can now easily see that $\Omega$ has a similar action on a momentum eigenstate:

$$
\begin{aligned}
\Omega|a\rangle_{p} & =\Omega \int e^{i x a / \hbar}|x\rangle_{x} \frac{d x}{\sqrt{2 \pi \hbar}} \\
& =\int e^{-i x a / \hbar}|x\rangle_{p} \frac{d x}{\sqrt{2 \pi \hbar}} \\
& =|a\rangle_{x}
\end{aligned}
$$


where in the middle step we use the anti-linearity of $\Omega$. Indeed, from this it follows that $\Omega^{-1}=\Omega$ and

$$
\begin{aligned}
\Omega \widehat{x} \Omega & =\widehat{p} \\
\Omega \widehat{p} \Omega & =\widehat{x} .
\end{aligned}
$$

We see immediately the action on the generators of the quantum torus:

$$
\begin{aligned}
& \Omega U \Omega=V^{-1}, \\
& \Omega V \Omega=U^{-1} .
\end{aligned}
$$

We can now readily calculate the action of $\Omega$ on different components of the propagator. For example,

$$
\begin{aligned}
\Omega E_{x} \Omega|a\rangle_{p} & =\Omega \int_{[0,1)+2 \mathbb{Z}}|x\rangle_{x x}\langle x \mid a\rangle_{x} d x \\
& =\int_{[0,1)+2 \mathbb{Z}} \delta(x-a)|x\rangle_{p} d x \\
& =E_{p}|a\rangle_{p} .
\end{aligned}
$$

This last equality is due to the fact that $x$ is really just a dummy variable being integrated over. Similarly, we find

$$
\Omega O_{x} \Omega=O_{p}, \quad \Omega L \Omega=B, \quad \Omega R \Omega=T .
$$

Also, since $\Omega$ takes $\widehat{x} \rightarrow \widehat{p}, i \rightarrow-i$, we find

$$
\Omega X^{-1 / 2} \Omega=Y^{1 / 2} \quad \Omega Y^{-1} \Omega=X, \quad \Omega S \Omega=S^{\dagger} .
$$

Thus on the propagator (eqn. 11),

$$
\begin{aligned}
\Omega F \Omega & =\Omega\left(E_{x}+X^{-1 / 2} O_{x}\right)\left(B+Y^{-1} T\right) S \Omega \\
& =\left(E_{p}+Y^{1 / 2} O_{p}\right)(L+X R) S^{\dagger} \\
& =S^{\dagger}(B+Y T)\left(E_{x}+X^{1 / 2} O_{x}\right) \\
& =F^{\dagger} .
\end{aligned}
$$

True to its classical origin, the full quantum propagator exhibits the appropriate time-reversal symmetry. What this means is that time-reversal symmetry 
holds at all values on the $\theta$-torus. We can see easily how to implement time reversal at the point $\theta=(0,0)$ :

$$
\begin{aligned}
\Omega \Phi_{m}^{(0,0)} & =\Omega\left(\frac{1}{\sqrt{N}} \sum_{k}\left|\frac{m}{N}+k\right\rangle_{x}\right) \\
& =\frac{1}{\sqrt{N}} \sum_{k}\left|\frac{m}{N}+k\right\rangle_{p} \\
& =\widetilde{\Phi}_{m}^{(0,0)}=\sum_{n=0}^{N-1}\left(\mathcal{F}^{N}\right)_{m n}^{-1} \Phi_{n}^{(0,0)} .
\end{aligned}
$$

Thus on the subspace $\mathcal{H}_{\hbar}(0), \Omega$ can be implemented via a matrix Fourier transform combined with complex conjugation. This is precisely the form used in ref. ${ }^{-}$to demonstrate time-reversal symmetry for the Balazs-Voros matrices.

\section{Acknowledgements}

The authors would like to thank Andrew Lesniewski, Christopher King, Lev Kaplan, Eric Heller, Jon Tyson and Sidney Coleman for many fruitful discussions. The authors would also like to thank the referee for insightful and constructive comments which we hope have made the paper more readable. 


\section{References}

[1]1. N. Balazs and A. Voros: Quantized Baker's Transformation, Ann. Phys., 190, 1-31 (1989)

[2]2. M. Saraceno: Classical Structures in the Quantized Baker's Transformation, Annals of Physics 199, 37-60 (1990)

[3]3. R. Rubin and N. Salwen: The Baker's Map on the $\theta$-Torus, manuscript in progress

[4]4. A. Lesniewski, R. Rubin, and N. Salwen: Classical Limits for Quantum Maps on the Torus, Journal of Math Phys, to appear

[5]5. S. Klimek, A. Lesniewski, N. Maitra and R. Rubin: Ergodic Properties of quantized Toral Automorphisms, Harvard University preprint (1995)

[6]6. R. Rubin and A. Lesniewski: Quantum Mechanics on a Torus, manuscript in progress

[7]7. D. Esposti: Quantization of Orientation Preserving Automorphisms of the Torus, Ann. l'Inst. H. Poincare, 58, 323-341 (1993)

[8]8. S. De Bievre, D. Esposti and R. Giachetti: Quantization of a Class of Piecewise Affine Transformations of the Torus, Preprint (1994)

[9]9. S. Zelditch: Quantum Ergodicity for $C^{*}$-dynamical systems, Comm.Math.Phys. (to appear)

[10]10. K. Hepp: The Classical Limit for Quantum Mechanical Correlation Functions, Comm. Math. Phys. 35, 265-277 (1974)

[11]11. J.H. Hannay and M. Berry: Quantization of Linear Maps on a Torus - Fresnel Diffraction of a Periodic Grating, Physica, 1D, 267-290 (1980) 
Figure 1: The baker's map on the torus. The square gets squished to half its height and stretched to twice its length, and the right region gets chopped off and placed back on top.

Figure 2: One iteration of the classical baker covering map. Observe that all "LEFT" regions map to "BOTTOM" regions, and "TOP" to "RIGHT". In this way, acting on periodic functions the covering map is exactly the baker's map on the torus. 
Table 1

$\begin{array}{ccc}\text { Operator }(\mathcal{O}) & \left(x_{0}, p_{0} \in ?\right) & \lim _{\hbar \rightarrow 0} \| \mathcal{O}\left|\phi_{\hbar} ; x_{0}, p_{0}\right\rangle \| \\ E_{x} & \tilde{e}_{x} & 1 \\ E_{x} & \tilde{e}_{x} & 0 \\ O_{x} & \tilde{o}_{x} & 0 \\ O_{x} & \tilde{o}_{x} & 1 \\ E_{p} & \tilde{e}_{p} & 1 \\ E_{p} & \tilde{e}_{p} & 0 \\ O_{p} & \tilde{o}_{p} & 0 \\ O_{p} & \tilde{o}_{p} & 1 \\ L & \widetilde{l} & 1 \\ L & \widetilde{l} & 0 \\ R & \widetilde{r} & 0 \\ R & \widetilde{r} & 1 \\ B & \widetilde{b} & 1 \\ B & \tilde{b} & 0 \\ T & \widetilde{t} & 0 \\ T & \widetilde{t} & 1\end{array}$

